of the resulting equations is reduced by Fock's use of a preferred system of co-ordinates, which he calls 'harmonic'. Fock accords a particular significance to harmonic coordinates (each of which satisfies a linear, generally covariant, equation), apparently because a harmonic system is uniquely determined to within a Lorentz transformation, under suitable supplementary conditions.

The changes effected for the new edition are summed up in the author's preface as follows: "The second edition differs from the first by some additions and reformulations. The question of the uniqueness of the mass tensor is treated in more detail (Section $31 *$ ) and is illustrated by two examples (Appendices B and C). The notion of conformal space is introduced and used as a basis for the treatment of Einsteinian statics (Sections 56 and 57). Greatest care has been applied to the formulation of the basic ideas of the theory and to the elucidation of those points in which the author's views differ from the traditional (Einsteinian) ones. Thus, in order to discuss the general aspects of the relativity principle Section $49 *$ has been added".

Prof. Fock's unorthodox views, expounded with a forceful clarity, undoubtedly help to advance our understanding of the theory of gravitation, making this a work of major importance. Elizabeth HiltoN

\section{STELLAR STATISTICS}

\section{Statistical Astronomy}

(Dover Publication No. 301.) By Robert J. Trumpler and Harold F. Weaver. Pp. Xxi+644. (New York: Dover Publications Inc.; London: Constable and Co., Ltd., 1962.) 24s.; 3.00 dollars.

STATISTICAL Astronomy deals with statistical investi$\mathcal{S}$ gations of the system of stars, and concentrates on such problems as the space distribution of stars, the distribution of stars on the $H-R$ diagram, the distribution of stellar velocities, and the phenomenon of galactic rotation. The statistical techniques involved are generally elementary in principle and tedious in application, and are fully developed in the first part of the book.

On reading such a detailed account and development of the subject as is presented here, one cannot help feeling that statistical astronomy has absorbed a great amount of the energy of astronomers and produced singularly few results. The authors should be fully aware that, because of the existence of interstellar dust, any star-counts can only lead to a 'local' picture of the star system, and a very distorted picture at that, if allowance is made only for a 'smooth' distribution of dust clouds; yet the book spends more than 120 pages doing just that. Shapley's classical investigation of the globular clusters which first led to a correct assessment of the star system is not mentioned, presumably because it involved too little statistics. Again, the construction of $H \cdot R$ diagrams for stars in the solar noighbourhood involves much statistical manipulation and takes up a correspondingly large space in the book, yet it is nothing like as useful as the $H-R$ diagrams for star clusters, which involve little statistics and receive correspondingly brief treatment. In the field of stellar motions, the book gives a good description of Kapteyn's two-stream model and Schwarzschild's ellipsoidal model; it then develops the latter along Charlier's method of moments. The treatment is complete from an observational point of view, but the most intricate part of the mathematical problem (p. 66) is shirked by a facile reference to the two-dimensional case. It omits to mention Ambarzumian's method of determining the distribution of space motions without assuming a model. It seems that the best part of the book is the part dealing with the phenomenon of galactic rotation. Oort's original theory and Bottlinger diagram are set out very clearly, and are further developed in a sound manner. This subject is mainly kinematical and dynamical in character, and is only marginally statistical.

There is an unfortunate idea among astronomers that statistical astronomy is a dull subject, that nothing of value has come out of it that cannot be improved on by non-statistical means. This is not true. There are exciting problems in this field, and these problems can be nothing but statistical, to witness, the distribution of interstellar matter and the clustering of galaxies. It is a pity that the only book in English that bears the title of "statistical astronomy' fails to include these more exciting problems, and presents the subject in a rather unattractive manner by confining itself to problems in stellar astronomy. But, since there is also a dearth of text-books on stellar astronomy, this book is quite worth its Dover edition price from the point of view of the serious student.

T. KIANG

\section{A BIOLOGIST LOOKS AT MAN}

\section{Genetics and Man}

By Prof. C. D. Darlington. Revised edition of The Facts of Life. Pp. $382+8$ plates. (London: George Allen and Unwin, Ltd., 1964.) 42s. net.

$\mathrm{N}$ the preface of the first edition of his book The Facts I of Life, published in 1953, Darlington defined his purpose, "to show the immense possibilities which await the application of the elementary principles of heredity to the great problems of society". In the preface of the revised edition, retitled Genetics and Man, Darlington explains that it has been necessary to abridge the old text in order to make room for new material. Certainly the new title is more apt, because the volume does not contain much information about what are popularly considered to be the facts of life.

The first comment to make about this book is that it is most entertaining. Geneticists, particularly those concerned with human genetics, can complain that some of the arguments are developed by an oversimplification of the facts. The biologists may be annoyed by occasional lapses into teleology, "The fly therefore has to reduce recombination" (p.120). The stylists may appreciate the musical analogies, "mitosis is a symphony consisting of four movements" (p. 69), but find some of the constructions rather tortuous. The cynics will comment on a certain naivety in the approach to problems involving human sexual relationships. There are one or two places where the reader is arrested by prejudice, for example, in a discussion of the education of normal women, "Girls are therefore prepared for a life which most of them will never follow, or wish to follow, and which it would be disastrous to the community if they did follow" (p. 317). This appalling state of affairs is attributed to the great influence that the "positively homosexual type of women" (p. 316) has had on the educational system!

In order to develop the theme of genetic determinism, the reader is taken smoothly through the advances in genetics from Moses via Mendel to molecules. There are interesting excursions into the history of forgeries that disturbed the scientific world. Having laid the foundation, Darlington philosophizes on the importance of the genetic background in all human situations. Advances in medicine have so reduced the untreatable causes of morbidity and mortality that medical practitioners have become increasingly aware of the importance of the family history of their patients-if not their genes. So Darlington possibly overstates the case when he writes, "And, when medicine wakes up to the existence of biology and rediseovers a philosophy, the genetic individual will be restored to his place" (p. 243).

These criticisms do not, however, seriously detract from the merits of a book that can be read both for pleasure and profit by laymen and scientists. SyLvia D. LAwLER 